\title{
Ultrafast $\mathrm{Dy}^{3+}$ :fluoride fiber laser beyond $\mathbf{3} \boldsymbol{\mu m}$
}

\author{
Yuchen Wang, ${ }^{1,2, *}$ (1) Frédéric Jobin, ${ }^{3}$ (i) Simon Duval, ${ }^{3,4}$ Vincent Fortin, ${ }^{3}$ Paolo Laporta, ${ }^{1,2}$ \\ Martin Bernier, ${ }^{3}$ Gianluca Galzerano, ${ }^{1,2}$ and Réal Vallée ${ }^{3}$
}

\author{
${ }^{1}$ Dipartimento di Fisica, Politecnico di Milano, Piazza Leonardo da Vinci 32, Milano 20133, Italy \\ ${ }^{2}$ Istituto di Fotonica e Nanotecnologie-CNR, Piazza Leonardo da Vinci 32, Milano 20133, Italy \\ ${ }^{3}$ Centre d'optique, photonique et laser (COPL), Université Laval, Québec G1V OA6, Canada \\ ${ }^{4}$ Femtum inc., 1405 boul. Parc Technologique, 2nd floor, Québec G1P 4R1, Canada \\ ${ }^{*}$ Corresponding author: yuchen.wang@polimi.it
}

Received 16 October 2018; revised 6 December 2018; accepted 7 December 2018; posted 10 December 2018 (Doc. ID 346573); published 14 January 2019

\begin{abstract}
We report a passively mode-locked $\mathrm{Dy}^{3+}$ :fluoride fiber laser emitting around $3.1 \mu \mathrm{m}$ based on the nonlinear polarization evolution technique in a ring configuration, using in-band pumping at $2.8 \mu \mathrm{m}$. Transform-limited and selfstarting mode-locked pulses as short as $\mathbf{8 2 8} \mathrm{fs}$ with a center wavelength around $3.1 \mu \mathrm{m}$ and repetition rates up to $60 \mathrm{MHz}$ are obtained. In the single-pulse regime, a maximum average output power of $204 \mathrm{~mW}$ is measured, corresponding to a peak power of $4.2 \mathrm{~kW}$ and a pulse energy of $4.8 \mathrm{~nJ}$. This first demonstration, to the best of our knowledge, of a femtosecond mode-locked fiber laser emitting directly beyond $3 \mu \mathrm{m}$ paves the way for frequency comb synthesis in the molecular fingerprint region. (๑2019 Optical Society of America
\end{abstract}

https://doi.org/10.1364/OL.44.000395

Femtosecond mode-locked lasers have enabled numerous recent innovations in fundamental physics, photochemistry, metrology, spectroscopy, microscopy, and material processing. The equidistant mode structure of optical frequency comb synthesizers based on fully stabilized mode-locked lasers brings forth possibilities of performing real-time spectroscopic measurements with broad bandwidth, high precision and absolute frequency calibration [1]. Ultrafast mid-infrared (mid-IR) laser sources emitting within the atmospheric window of 3 to $5 \mu \mathrm{m}$ are of particular interest for remote sensing and spectroscopic applications because of for their ability to access the fundamental rotational-vibrational absorption features of many common gas-phase molecules (e.g., $\mathrm{CH}_{4}, \mathrm{CO}$, and $\mathrm{CO}_{2}, \mathrm{HCN}$.) [2]. Therefore, mid-IR femtosecond mode-locked lasers emitting beyond $3 \mu \mathrm{m}$ could enable a new class of mid-IR frequency comb sources that finds immediate application in molecular fingerprinting [3,4], trace gas detection for environmental monitoring [5], and breath analysis for medical diagnosis [6].

Currently, commercially available mid-IR frequency comb sources are mostly based on nonlinear downconversion techniques (e.g., difference frequency generators, optical parametric amplifiers, and oscillators) that are less efficient and more complex compared to mode-locked laser comb sources. This difficulty has been tackled by a novel class of mid-IR laser sources based on rare-earth-doped fluoride fibers that have extended the reach of femtosecond lasers into the mid-IR region around $3 \mu \mathrm{m}$. First, experimental works based on Er- and Hodoped fluoride fibers have demonstrated mode-locking operation with pulse durations at a picosecond level, employing fast saturable absorbers such as a semiconductor saturable absorber mirror $[7,8]$ and graphene [9]. More sophisticated Er- and Ho-doped fluoride fiber systems based on the nonlinear polarization evolution (NPE) technique were been demonstrated later on, shortening the pulse duration down to the femtosecond regime [10,11].

$\mathrm{Dy}^{3+}$-ion-doped fluoride (e.g., fluorozirconate and fluoroindate glasses) fibers are increasingly attractive for efficient generation of light beyond $3 \mu \mathrm{m}$, as they show a broader emission cross section with a peak around $2.94 \mu \mathrm{m}$ as compared to the more common $\mathrm{Er}^{3+}$ and $\mathrm{Ho}^{3+}$-doped fluoride fibers (peaking, respectively, at 2.75 and $2.85 \mu \mathrm{m}$ ). These spectroscopic properties of $\mathrm{Dy}^{3+}$-doped fluoride fibers could be interpreted as a potential of supporting shorter pulses when the gain linewidth is fully utilized, and the intracavity dispersion is properly compensated [12]. The broad emission bandwidth has been confirmed by its wide continuous-wave (CW) emission wavelength tunability from 2.8 to $3.4 \mu \mathrm{m}$ in Ref. [13]. Theoretical modeling of the in-band pumped Dy laser system demonstrated also the possibility of gain switching regime with output pulse energies of tens of microjoules [14]. Recently, the active mode-locking of a $\mathrm{Dy}^{3+}$ :fluoride fiber laser has been reported, generating 33 ps pulses tunable from 2.97 to $3.3 \mu \mathrm{m}$ [15]. Despite these impressive results, much improvement in terms of efficiency and pulse duration are expected for mode-locked $\mathrm{Dy}^{3+}$ :fluoride fiber lasers.

In this Letter, we demonstrate a femtosecond $\mathrm{Dy}^{3+}$ :fluoride fiber ring laser based on the NPE mode-locking technique. With an efficient in-band pumping scheme, stable and selfstarting pulses with a duration of 828 femtosecond are generated at repetition rates up to $60 \mathrm{MHz}$, with a maximum peak power of $4.2 \mathrm{~kW}$. To the best of our knowledge, this is the first demonstration of direct generation of femtosecond pulses 


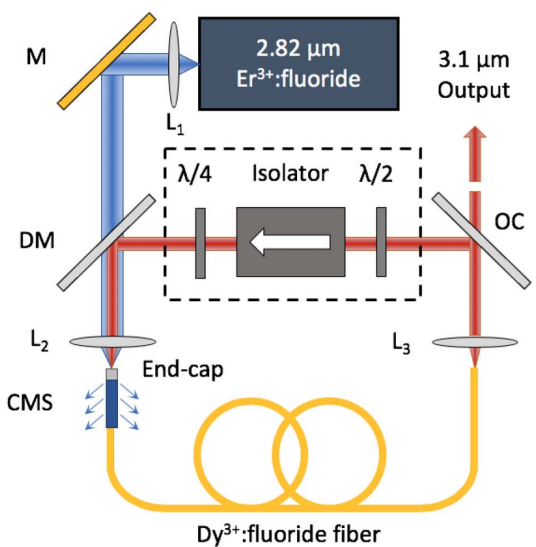

(a)

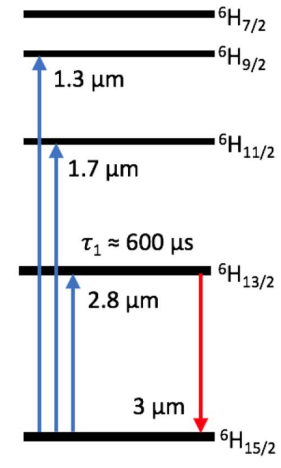

(b)
Fig. 1. (a) Schematic diagram of the ring fiber laser cavity and (b) simplified energy level diagram of a $\mathrm{Dy}^{3+}$ ion. $\mathrm{M}$, protected gold mirror; DM, dichroic mirror; CMS, cladding mode stripper; OC, output coupler; $\lambda / 4$, quarter-wave plate; $\lambda / 2$, half-wave plate; $L_{1,2,3}$, aspheric $\mathrm{ZnSe}$ lenses $\left(\mathrm{f}=12.5 \mathrm{~mm}\right.$ ); $\tau_{1}$, laser upper state lifetime.

above $3 \mu \mathrm{m}$ from a fiber laser resonator, showing the great potential of fluoride fiber lasers for ultra-short pulse generation further into the mid-IR.

The laser setup in this experiment is schematically illustrated in Fig. 1(a). Single-mode $\mathrm{Dy}^{3+}$-doped fluorozirconate glass fibers (Le Verre Fluoré) with lengths of 3 and $4.5 \mathrm{~m}$ are used as the active medium. They have a $\mathrm{Dy}^{3+}$-ion doping concentration of $0.2 \mathrm{~mol} \%$, a core diameter of $12.5 \mu \mathrm{m}$, a cladding diameter of $125 \mu \mathrm{m}$, and a numerical aperture (NA) of 0.16 . The population on the ${ }^{6} \mathrm{H}_{15 / 2}$ ground state is excited into the ${ }^{6} \mathrm{H}_{13 / 2}$ first upper state using an in-band pumping scheme [see Fig. 1(b)]. A homemade diode-pumped $\mathrm{Er}^{3+}$ :fluoride fiber laser based on a design similar to Ref. [16], operating at $2.825 \mu \mathrm{m}$ with a maximum CW output power of $10 \mathrm{~W}$, is used as the pump source in this experiment. At $2.825 \mu \mathrm{m}$, an unsaturated absorption coefficient of $39 \mathrm{~dB} / \mathrm{m}$ can be calculated from the absorption cross section of Dy-doped fluoride glasses [17]. The pump beam delivered through a single-mode fiber with a core diameter of $15 \mu \mathrm{m}$ is collimated by an aspheric $\mathrm{ZnSe}$ lens ( $\mathrm{f}=12.5 \mathrm{~mm}$ ). Inside the resonator, a pair of identical antireflection-coated plano-convex aspheric $\mathrm{ZnSe}$ lenses ( $\mathrm{f}=12.5 \mathrm{~mm}$ ) is used for fiber beam collimation and reinjection. A pump dichroic mirror (DM) and an output coupler (OC) are positioned with $45^{\circ}$ angles at the two ends of the fiber to close the ring cavity. For unpolarized light, the $45^{\circ} \mathrm{DM}$ has a transmittance $\left(\mathrm{T}_{\mathrm{DM}}\right)$ of $54 \%$ at $2.825 \mu \mathrm{m}$ and a reflectance $\left(\mathrm{R}_{\mathrm{DM}}\right)$ larger than $99 \%$ at $3.1 \mu \mathrm{m}$; the $45^{\circ} \mathrm{OC}$ has a $\mathrm{T}_{\mathrm{OC}}$ of $22.5 \%$ at $3.1 \mu \mathrm{m}$. The multilayer dielectric coatings on these components are fabricated using in-house coating facilities. The free-space path inside the cavity has a length of $39 \mathrm{~cm}$.

Particular attention is devoted to the protection of the fiber end-face at the pump injection side. Issues related to $\mathrm{OH}^{-}$diffusion and a thermal runaway at high power may lead to fiber tip degradation and damage under prolonged operation [18], as the pumping wavelength of $2.82 \mu \mathrm{m}$ is very close to the water absorption band. This problem is effectively mitigated by splicing the fluoride fiber with a short $(<100 \mu \mathrm{m})$ segment of $\mathrm{AlF}_{3}$ fiber (coreless, $125 \mu \mathrm{m}$ diameter) which serves as a protective end-cap (with Vytran GPX-3000 fiber splicer). Due to the relatively small core dimension, both the pump injection and the cavity are very sensitive to misalignment. A cladding mode stripper (CMS) with a refractive index varying from 1.49 to 1.52 is implemented to gradually remove the residual pump power in the fiber cladding.

To achieve passive mode locking through NPE in the active fiber, a polarization control stage (the section in the dashed rectangle in Fig. 1) is implemented in the free-space segment of the resonator and composes a polarizing optical isolator, a zeroorder quarter-wave plate, and a zero-order half-wave plate, all designed for a $3.2 \mu \mathrm{m}$ wavelength. The optical isolator (Thorlabs IO-4-3200-WG) ensures unidirectional laser propagation in the ring cavity. The counter-clockwise ring laser propagation direction (as depicted in Fig. 1) is selected to have co-propagating pump and laser beams, which maximizes the nonlinear polarization rotation effect and facilitates the self-starting operation of the laser in a mode-locked state.

By carefully adjusting the combinations of the wave plate orientation, mode-locking operation is achieved with fiber lengths of 3 and $4.5 \mathrm{~m}$, showing different spectral and temporal characteristics. For shorter fiber lengths, mode locking was not achieved, since the emission wavelength reduced to $\sim 3 \mu \mathrm{m}$, where the intracavity elements show significantly higher losses. The mode-locking incident pump power thresholds are 1.7 and $1.8 \mathrm{~W}$ for 3 and $4.5 \mathrm{~m}$ fibers, respectively. A temporal trace of the mode-locking pulse train with a fiber length of $4.5 \mathrm{~m}$ measured with a pre-amplified liquid-nitrogen cooled $\mathrm{HgCdTe}$ photodiode (Kolmar Technologies KV104-0.1-E/ $\mathrm{KA}, 500 \mathrm{MHz}$ bandwidth) is shown in Fig. 2(a). The temporal signal suggests a single-pulse operation without Q-switching instabilities. This is confirmed by its normalized power spectral density measured by an electrical spectrum analyzer shown in Fig. 3. Over the recorded span of $1 \mathrm{GHz}$, the fundamental and harmonic components of the repetition frequency have no observable sidebands at a relative power level down to $-80 \mathrm{~dB}$ at a resolution bandwidth (RBW) of $1 \mathrm{MHz}$ [Fig. 3(a)].
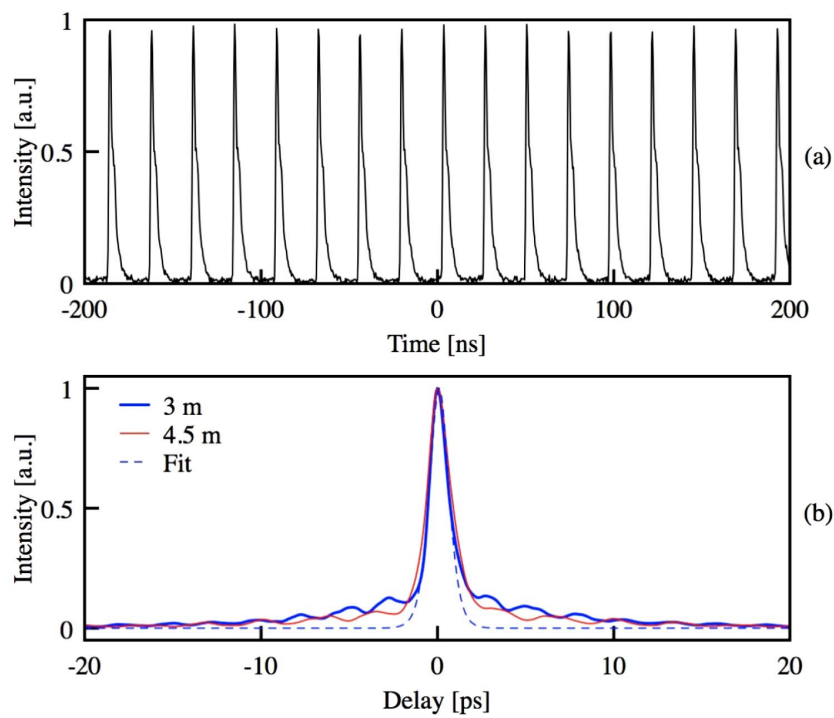

Fig. 2. (a) Temporal trace of the mode-locked pulse train (with $4.5 \mathrm{~m}$ fiber); (b) pulse autocorrelation traces for different fiber lengths ( $3 \mathrm{~m}$ in blue, $4.5 \mathrm{~m}$ in red) and a hyperbolic secant fit (in blue dashed line) for the shortest case with $3 \mathrm{~m}$ fiber. 

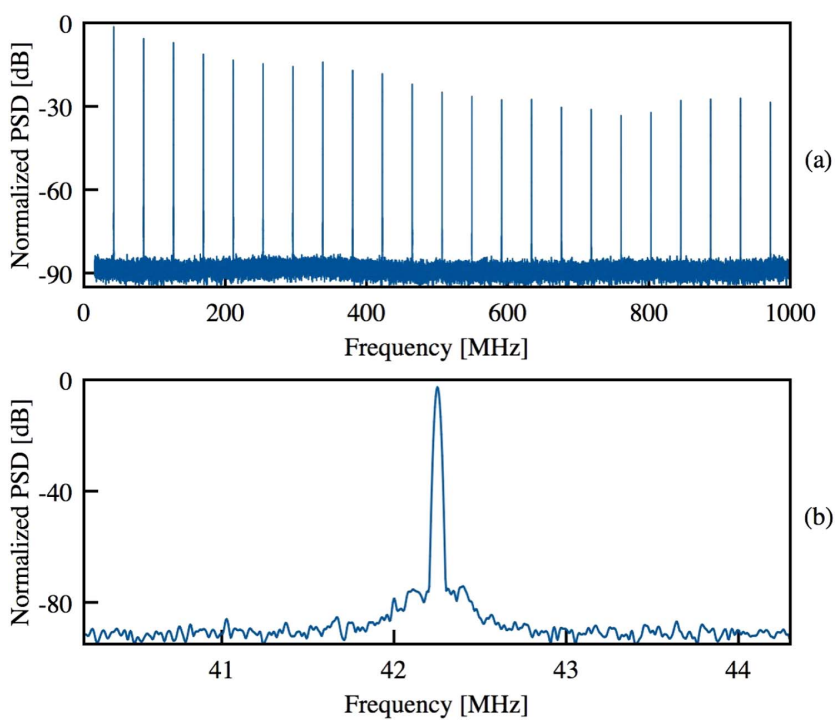

Fig. 3. Normalized power spectral density of the pulse train signal (a) over a $1 \mathrm{GHz}$ span (1 MHz RBW) and (b) at around the fundamental repetition frequency of $42.2 \mathrm{MHz}$ at $20 \mathrm{kHz} \mathrm{RBW}$ (4.5 $\mathrm{m}$ fiber).

With a finer frequency resolution of $20 \mathrm{kHz}$ [Fig. 3(b)], the fundamental repetition frequency shows a signal-to-noise ratio over $85 \mathrm{~dB}$, with weak modulations $(-70 \mathrm{~dB}$ relative level) most likely related to technical noises (e.g., vibrational and acoustic environmental disturbances and pump intensity noise) acting on pulse train intensity and its repetition rate. The fundamental pulse repetition frequencies are measured to be 59.9 and $42.2 \mathrm{MHz}$ for the cavity configurations with fiber lengths of 3 and $4.5 \mathrm{~m}$, respectively.

Intensity autocorrelations are measured with an autocorrelator (Femtochrome FR-103XL/IR/1700) to evaluate the temporal profile of the pulses. The traces for the two different fiber lengths are shown in Fig. 2(b). The pulse autocorrelation of the $3 \mathrm{~m}$ fiber configuration fits fairly well with the autocorrelation of a squared hyperbolic secant function [shown as dashed lines in the Fig. 2(b)]. Full-width at half-maximum (FWHM) pulse widths of 828 and $1010 \mathrm{fs}$ are retrieved from the autocorrelations with fiber lengths of 3 and $4.5 \mathrm{~m}$, respectively (724 and 883 fs from the sech ${ }^{2}$ fit). The observable pedestals in the autocorrelation shape are most likely due to the strong dispersive waves corresponding to intense Kelly sidebands in the pulse optical spectrum (see Fig. 4).

An optical spectrum of a single-pulse mode-locking condition generated with a $4.5 \mathrm{~m}$ fiber, measured with an optical spectrum analyzer (OSA) (Yokogawa AQ6375L) at $0.2 \mathrm{~nm}$ resolution, is shown in Fig. 4. Centered at $3083 \mathrm{~nm}$, the solitonic bell shape has a FWHM of $13.7 \mathrm{~nm}(0.43 \mathrm{THz})$. The pulse spectrum has the typical shape of mode-locked fiber lasers operating in an anomalous dispersion regime. The slight modulations over the spectrum are due to ambient water vapor absorptions. In the case of the $3 \mathrm{~m}$ fiber, the spectrum is centered at a shorter wavelength of $3050 \mathrm{~nm}$ with a FWHM of $14.7 \mathrm{~nm}(0.47 \mathrm{THz})$. Indeed, for in-band pumping in a quasi-three-level laser scheme, the gain profile and emission wavelength are intrinsically dependent on the fiber length. Observable in both cases are the intense Kelly sidebands that

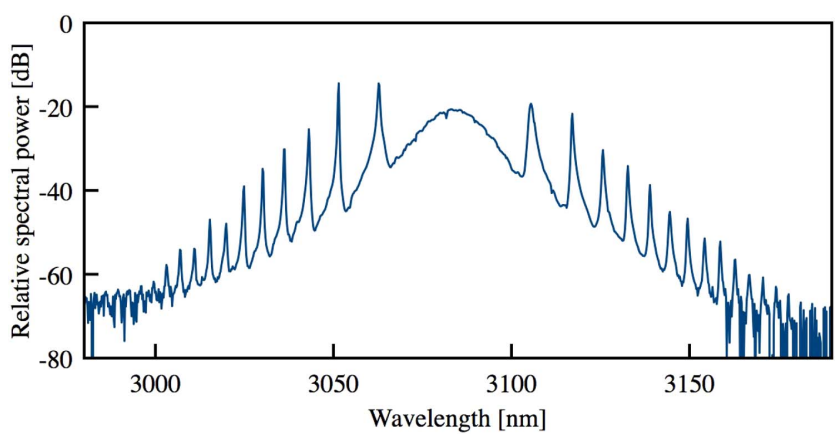

Fig. 4. Optical spectrum of the mode-locking pulse train (generated with $4.5 \mathrm{~m}$ fiber) measured with the OSA at $0.2 \mathrm{~nm}$ resolution.

accompany the soliton spectrum. The physical mechanisms behind the generation of these sidebands first include the linear and nonlinear phase delays and their phase-matching condition that determines the wavelengths of the sidebands and, secondly, the large intracavity periodic variations of the soliton amplitude induced by the gain modulation inside the long active fiber and the discrete losses in the free-space optical components that exacerbate the amplitude of the sidebands [19].

Considering the estimated pulse durations, time-bandwidth products (TBWPs) of the pulse trains generated with 3 and $4.5 \mathrm{~m}$ of fibers are evaluated to be 0.39 and 0.43 , respectively. Therefore, the pulses are close to the transform limit (TBWP $=0.315$ for sech $^{2}$ pulse).

Continuous mode-locking operation was routinely achieved, without any thermal control, over periods longer than $1 \mathrm{~h}$ with a long-term stability better than $10 \%$. Over a shorter observation time of $10 \mathrm{~min}$, the fractional RMS fluctuation reduces to $0.6 \%$ (measured with Gentec XLP12-3S power detector), showing that temperature variations might be the main cause of long-term power fluctuations. With $\mathrm{T}_{\mathrm{OC}}=22.5 \%$, the average output powers measured in the single-pulse regime are 66 and $72 \mathrm{~mW}$ with cavity configurations using 3 and $4.5 \mathrm{~m}$ fibers, respectively. In order to optimize the overall laser efficiency, a larger output coupling $\mathrm{T}_{\mathrm{OC}}=55 \%$ was also tested, with experimental results showing similar pulse optical spectrum and pulse durations. In this configuration, a maximum average output power of $204 \mathrm{~mW}$ at $2.2 \mathrm{~W}$ incident pump power is obtained with a $4.5 \mathrm{~m}$ fiber $(175 \mathrm{~mW}$ with $3 \mathrm{~m}$ fiber at $2.1 \mathrm{~W}$ incident pump power) in a single-pulse regime. Thanks to the efficient pumping scheme, in a mode-locking regime, the optical-to-optical efficiency is larger than $10 \%$. For 3 and $4.5 \mathrm{~m}$ of fibers, in stable mode-locking regimes, maximum pulse peak powers of 3.11 and $4.21 \mathrm{~kW}$ are estimated, which correspond to 2.92 and $4.83 \mathrm{~nJ}$ of pulse energy, respectively.

During mode-locking operation, we also noted that the soliton central wavelength can be continuously tuned over a range wider than $40 \mathrm{~nm}$ by slightly rotating the quarter-wave plate. The mechanism for this wavelength tuning remains to be investigated in further experiments. As a preliminary hypothesis, it might be due to the interplay between the polarization and wavelength-dependent reflectivities of the OC and the DM, and the wavelength dependence of the wave plate delays, effectively acting as a tunable spectral filter. Dependence of the mode-locking soliton peak wavelength on the pump power level 
was not experimentally observed in the narrow pump power range allowing the mode-locking operation through NPE.

In the current configuration, the mode-locking regime is characterized by a strong anomalous intracavity dispersion that needs to be compensated for to reach the minimum pulse duration [12]. With measured spectra, estimations of the fiber dispersion parameter $D$ at different wavelengths, can be derived, employing the following relation [19]:

$$
D=\frac{2 \lambda_{c}^{2}}{c l m}
$$

where $l$ is the fiber length, $\lambda_{c}$ is the center wavelength, $c$ is the speed of light, and $m$ represents the slope of the least square linear fit of the squared wavelength offsets $(\Delta \lambda)^{2}$ of the $N$ th sideband from the center wavelength with respect to the sideband order $N$, as $m \approx(\Delta \lambda)^{2} / N$. From the measured spectrum of the $4.5 \mathrm{~m}$ fiber configuration shown in Fig. 4, neglecting the dispersion contributions of the intracavity free-space optical components, an estimated fiber dispersion parameter of $23.7 \mathrm{ps} \mathrm{nm}^{-1} \mathrm{~km}^{-1}$ at the center wavelength of $3083 \mathrm{~nm}$ is retrieved, which is in good agreement with the bulk fluoride material of $22.3 \mathrm{ps} \mathrm{nm}^{-1} \mathrm{~km}^{-1}$ [20]. For the $3 \mathrm{~m}$ fiber, the dispersion parameter at $3050 \mathrm{~nm}$ is estimated to be $22.9 \mathrm{ps} \mathrm{nm}^{-1} \mathrm{~km}^{-1}$. The total group delay dispersion (GDD) for the $4.5 \mathrm{~m}$ fiber is then estimated to be around $-0.538 \mathrm{ps}^{2}$ at $3083 \mathrm{~nm}$, which leads to a theoretical solitonic pulse duration of $\sim 1$ ps according to the soliton "area theorem" [12]; this is in good agreement with the experimental results. This large amount of anomalous dispersion would require a normal dispersion element to be introduced into the resonator resulting in an anomalous net GDD close to zero for generating the shortest possible mode-locked pulses allowed by the available Dy gain bandwidth.

Compared to the demonstrated mode-locking performances of Er- and Ho-doped fluoride fiber lasers, Dy-based sources have some aspects such as pulse duration and output power that need optimizations. In the near future, better mid-IR coatings for the critical intracavity components and revised resonator designs should further increase the laser efficiency and operation spectral bandwidth; a careful dispersion compensation should bring the pulse duration down to sub-100 fs level. Thanks to the broad gain bandwidth and the simple quasithree-level laser scheme, longer fiber lengths could be adopted for operation at longer wavelengths. Preliminary experimental results with a $5.5 \mathrm{~m}$ long fiber showed a mode-locking central wavelength of $3183 \mathrm{~nm}$ with a similar pulse duration of $\sim 1$ ps. Together with the soliton self-frequency shift effect, ultrashort pulses could be pushed deeper into the mid-IR passively or in an in-amplifier configuration [21]. Moreover, by means of chirped-pulse optical amplification [22] in $\mathrm{Dy}^{3+}$ :fluoride fiber, and supercontinuum generation in fluoride fibers [23] or chalcogenide fibers [24], coherent mid-IR optical frequency combs could be potentially realized in a compact and portable all-fiber format, capable of performing high accuracy and highresolution molecular spectroscopy over the $2-5 \mu \mathrm{m}$ spectral region.

In conclusion, we have presented a self-starting passively mode-locked $\mathrm{Dy}^{3+}$ :fluoride fiber ring laser operating near
$3.1 \mu \mathrm{m}$ with a pulse duration of $828 \mathrm{fs}$, average output power higher than $200 \mathrm{~mW}$, and maximum pulse peak power exceeding $4 \mathrm{~kW}$, at repetition rates from 42 to $60 \mathrm{MHz}$. This first demonstration, to the best of our knowledge, of a fs modelocked fiber laser beyond $3 \mu \mathrm{m}$ further extends the reach of rare-earth-doped fluoride fiber lasers into the mid-IR, opening new possibilities for the direct synthesis of mid-IR frequency comb sources covering the molecular fingerprint region.

Funding. Natural Sciences and Engineering Research Council of Canada (NSERC) (IRCPJ469414-13); Fonds de Recherche du Québec-Nature et Technologies (FRQNT) (144616); Canada Foundation for Innovation (CFI) (5180); Regione Lombardia (n.19363/RCC); Ministero dell'Istruzione, dell'Università e della Ricerca (MIUR) (ESFRI RoadmapExtreme Light Infrastructure (ELI)).

\section{REFERENCES}

1. R. Holzwarth, T. Udem, T. W. Hänsch, J. C. Knight, W. J. Wadsworth, and P. St. J. Russell, Phys. Rev. Lett. 85, 2264 (2000).

2. A. Schliesser, N. Picqué, and T. W. Hänsch, Nat. Photonics 6, 440 (2012).

3. N. Coluccelli, M. Cassinerio, B. Redding, H. Cao, P. Laporta, and G. Galzerano, Nat. Commun. 7, 12995 (2016).

4. A. Gambetta, M. Cassinerio, D. Gatti, P. Laporta, and G. Galzerano, Sci. Rep. 6, 35541 (2016).

5. F. Adler, P. Masłowski, A. Foltynowicz, K. C. Cossel, T. C. Briles, I. Hartl, and J. Ye, Opt. Express 18, 21861 (2010).

6. C. Wang and P. Sahay, Sensors 9, 8230 (2009).

7. T. Hu, D. D. Hudson, and S. D. Jackson, Opt. Lett. 39, 2133 (2014).

8. A. Haboucha, V. Fortin, M. Bernier, J. Genest, Y. Messaddeq, and R. Vallée, Opt. Lett. 39, 3294 (2014).

9. C. Wei, X. Zhu, F. Wang, Y. Xu, K. Balakrishnan, F. Song, R. A. Norwood, and N. Peyghambarian, Opt. Lett. 38, 3233 (2013).

10. S. Duval, M. Bernier, V. Fortin, J. Genest, M. Piché, and R. Vallée, Optica 2, 623 (2015)

11. R. I. Woodward, D. D. Hudson, A. Fuerbach, and S. D. Jackson, Opt. Lett. 42, 4893 (2017).

12. H. A. Haus, IEEE J. Sel. Top. Quantum. Electron. 6, 1173 (2000).

13. M. R. Majewski, R. I. Woodward, and S. D. Jackson, Opt. Lett. 43, 971 (2018).

14. M. C. Falconi, D. Laneve, M. Bozzetti, T. T. Fernandez, G. Galzerano, and F. Prudenzano, J. Lightwave Technol. 36, 5327 (2018).

15. R. I. Woodward, M. R. Majewski, and S. D. Jackson, in CLEO Pacific Rim Conference (2018), paper PDP.8.

16. V. Fortin, M. Bernier, S. T. Bah, and R. Vallée, Opt. Lett. 40, 2882 (2015).

17. L. Gomes, A. F. H. Librantz, and S. D. Jackson, J. Appl. Phys. 107, 053103 (2010).

18. N. Caron, M. Bernier, D. Faucher, and R. Vallée, Opt. Express 20, 22188 (2012).

19. M. L. Dennis and I. N. Duling, IEEE J. Quantum Electron. 30, 1469 (1994).

20. F. Gan, J. Non-Cryst. Solids 184, 9 (1995)

21. S. Duval, J.-C. Gauthier, L.-R. Robichaud, P. Paradis, M. Olivier, V. Fortin, M. Bernier, M. Piché, and R. Vallée, Opt. Lett. 41, 5294 (2016).

22. M. E. Fermann and I. Hartl, Nat. Photonics 7, 868 (2013).

23. X. Jiang, N. Y. Joly, M. A. Finger, F. Babic, G. K. L. Wong, J. C. Travers, and P. St. J. Russell, Nat. Photonics 9, 133 (2015).

24. U. Møller, Y. Yu, I. Kubat, C. R. Petersen, X. Gai, L. Brilland, D. Méchin, C. Caillaud, J. Troles, B. Luther-Davies, and O. Bang, Opt. Express 23, 3282 (2015). 